\title{
Exploring the Experiences of Living with Infertility in Menopausal Women in Iran, A Qualitative Study
}

\begin{abstract}
Background: The qualitative studies are more effective in human experience than quantitative research. Do not have study focusing on the experience of menopausal infertile women who haven't experienced of motherhood in all their lives. The purpose of this qualitative study was to describe the experience of infertility in menopausal women.
\end{abstract}

Materials and methods: In a phenomenological study, conducted 17 semi-structured individual interviews in Ilam, west of Iran, in 2014. Study participants were selected purposively by using a snowball sampling technique. The participants met the inclusion criteria, including postmenopausal women with no history of pregnancy, at least one year after the start of menopause. All women with abnormal menopause such as menopausal induced by surgery, drugs or chemotherapy were excluded of study. Data analysis was carried out based the Colaizzi process for phenomenological data analysis in 8 steps, including; prepare the data, the meaning units, text encoding, revision of the text codes, categories based on similarity and proportion of classes, revision class and compare this data to ensure the reliability of the code, identification of themes reflecting the depth and comparison with other classes and report findings.

Result: Through analysis six sub themes emerged, including: physical factors, psychological factors, beliefs and religion, social relationships, instability in life and strategies. These were encompassed in the core category of 'motherhood experience'.

Conclusion: An identification experience of infertility is essential to develop appropriate consulting services. Due to the impact of various factors on the experience of infertile women, similar studies are recommended in different cultures.

Keywords: experiences of infertility, phenomenological study, qualitative research
Volume 5 Issue 3 - 2017

\author{
Ashraf Direkvand-Moghadam,' Mosayeb \\ Mozafari $^{2}$ \\ 'Faculty of Nursing and Midwifery, llam University of Medical \\ Sciences, Iran \\ ${ }^{2}$ Department of Nursing and Midwifery, llam University of \\ Medical Sciences, Iran
}

Correspondence: Ashraf Direkvand-Moghadam, Ph.D in Reproductive Health, Faculty of Nursing and Midwifery, llam University of Medical Sciences, Ilam, Iran, Tel/Fax + 00988432 2404 04, Email direkvand-a@medilam.ac.ir

Received: June 19, 2017 | Published: July 03, 2017

\section{Introduction}

More than 70 million infertile couples lived in the worldwide. The majority of infertile couples are residents in developing countries. These residents experience more negative consequences of infertility in compared with western societies. ${ }^{1}$ Motherhood is an important part of life in nearly all cultures. It is presented as an essential role for all Muslim women, especially Iranian women. ${ }^{2}$ Determining the actual global prevalence of infertility is difficult. However, nearly, $80-90 \%$ of couples have successfully conceived after 1year and $95 \%$ after 2 years, ${ }^{3}$ but also, one of four couples is affected by infertility in developing countries. ${ }^{4}$ The prevalence of primary and secondary infertility is $0.6 \%-3.4 \%$ and $10.5 \%$ respectively. ${ }^{5}$ The trends of women infertility have remained similar from 1990 to 2010 and the incidence of infertility is expected to increase to 7.7 million by $2025 .{ }^{4}$ In most Islamic societies, childbearing is a religious duty ${ }^{6}$ and infertile women face many challenges in their lives. Infertile women are uncomfortable in comparing infertile men. ${ }^{6}$ Based the results of studies, there are several consequences of infertility, including: societal repercussions, personal suffering, marital discord, psychological disorders, ${ }^{7,8}$ sexual dysfunctions. ${ }^{9,10}$ In the recent years, the life expectancy has increased due to improve the health, nutrition and health services. So that 5 million postmenopausal women will live in Iran in 2021."
Post-menopausal period is an important part of the woman's life, in which women experience some or all of the symptoms and complications. Some symptoms such as irritability, anger and feelings of depression increased around the time of menopause. It is estimated $26-33 \%$ of women experience the first episode of depression in their menopausal period. ${ }^{12}$ In menopausal period the decline in estrogen levels leads to a broad range of symptoms, including vasomotor symptoms such as hot flashes and night sweats. Other menopausal symptoms may include dizziness, tachycardia, irregular heart, atrophy of the vaginal mucosa and irritability bladder, mood changes, sleep disturbances, headaches, muscle pain, joint pain, difficulty concentrating and memory disorders. ${ }^{13}$ Several studies have been reported the relationship between reproductive history and menopausal symptoms. ${ }^{14-16}$ A study in France showed the relationship between multi-parity and decrease the risk of menopausal symptoms in women aged 40-65years. ${ }^{16}$ Another study reported the risk of hot flash is lower in Japanese women with higher number of live births. ${ }^{14}$ Nelson et al., ${ }^{15}$ reported that risk of severe vaginal dryness increased in infertile women during the menopausal transition. Also, women with a history of infertility experience the lower libido.$^{15}$ In contrast, another study reported that infertile women may have fewer hot flash episodes. ${ }^{17}$

It seems that the qualitative studies are more effective in human experience than quantitative research. ${ }^{18}$ In fact, the knowledge derived 
from qualitative research is not to determine a causal relationship. As far as we know, several qualitative researches have been done with the experience of infertility in women during reproductive ages. Other qualitative researches evaluated the menopausal experience in Iranian women who have experienced the motherhood. But we couldn't find study focusing on the experience of menopausal infertile women who haven't experienced of motherhood in all their lives. Hence the aim of the present qualitative study was to report the experience of infertility in menopausal women.

\section{Material and methods}

This qualitative study is a PhD thesis (Grant Number: 909279) on the post-menopausal infertile women's experiences. The researchers used two main paradigms of positivism and naturalism in scientific studies. In the positivistic paradigm, the existence of the phenomenon is in question if could it not objectively measure. Since the positivistic paradigm is not sufficient to react to the human experience, therefore, to meditate and understand the human experience of phenomena, using naturalistic paradigm is important. The qualitative research is exploratory in nature and uses words instead of numbers. ${ }^{18}$ Thus, the present study was performed as phenomenology study. We have conducted 17 semi-structured individual interviews in Ilam, west of Iran, in 2014 to achieve the experience of the participants. Study participants were selected purposively by using a snowball sampling technique through professional and social networking. The participants met the inclusion criteria, including postmenopausal women with no history of pregnancy, at least one year after the start of menopause. All women with abnormal menopause such as menopausal induced by surgery, drugs or chemotherapy were excluded of study. In the qualitative studies, the sample size of the study is not intended. Therefore, in the present study, sampling was performed until data saturation. ${ }^{19}$

\section{Data collection and analysis}

Semi-structured interviews were used to collect the data. The importance and purpose of the study were explained to the participants before the interview. All participants were free to withdraw from the study at any time and ethical points. Interviewer considers the confidentiality and privacy of the participants. Data were generated by using digitally recorded interviews in the home or workplace, according to the willingness of the participants. Each participant was separately interviewed and all conversations were taped. Interviews were at least 25 minutes and up to 65 minutes. The interview started with the questions, "please tell me about your experiences as an infertile woman, is infertility affected on your physical and psychological status? Is your physical and psychological status changed before and after the your menopause? ". Follow-up questions were designed to better explain the concepts and sometimes based on concepts derived from previous interviews.

Data analysis was concurrent with data collection. Data collection continued until the data saturation was reached. The interviews were transcribed into text by the interviewer shortly after it was conducted. Data analysis was carried out based the Colaizzi process for phenomenological data analysis in 8steps, including. ${ }^{20,21}$

i. Prepare the data (type of interviews).

ii. The meaning units (specific words, sentences or paragraphs from the speech of participants). iii. Text encoding (converting units of semantic tags, which reflect a summary of selected units).

iv. Revision of the text codes (over again, comparing the similarities and differences between the codes and then integrate the same code).

v. Categories based on similarity and proportion of classes.

vi. Revision class and compare this data to ensure the reliability of the code.

vii. Identification of themes reflecting the depth.

viii. Comparison with other classes and report findings.

Credibility, dependability, transferability and Conformability of the data were checked. Long-term involvement of researcher with the data subject, member check and peer check were used as potential contributor to data credibility. ${ }^{20}$ The recorded and codes interviews were assigned to 3 researchers with experience in qualitative research. Finally, their comments were used. Immersion of researcher in the data by recurrent reading of the transcript and constant comparability of significant information was used to enhance the dependability of the data. All participants' statements were presented without modification in order to increase the transferability. Also the demographic participants' characteristics reported on the details to readers. Demographic characteristics of participants in the interviews are presented in Table 1. Using multiple researchers on data analysis was used to ensure the data Conformability.

Table I Demographic characteristics of participants in the interviews

\begin{tabular}{lll}
\hline Variable & & \\
\hline Age (years)* & $52 \pm 2.88$ & \\
Duration of infertility* & $34 \pm 2.1$ & \\
$\begin{array}{l}\text { Duration of infertility } \\
\text { treatmeants* }\end{array}$ & $19 \pm 4$ & $12(70.58)$ \\
Occupation** & Housekeeper & $5(29.42)$ \\
& Employed & $6(35.2)$ \\
& Illiterate & $7(41.18)$ \\
Education level** & Elementary & \\
& Secondary school & $4(23.53)$ \\
\hline
\end{tabular}

*Mean \pm SD

**N (\%)

\section{Ethical consideration}

The study was approved by the Ethical Committee of Ilam University of Medical Sciences (EC.93.H.260). Participants provided written or oral informed consent upon recruitment as well as a process consent, which was used throughout the interviews. Confidentiality and privacy were ensured.

\section{Results}

With analysis the data, the motherhood is the main theme 
of life experience in menopausal infertile women. It seems that physical factors, psychological factors, beliefs and religion, social relationships, instability in life and strategies are more attention than other sub theme in all participants (Tables 2) (Table 3). In the physical sub theme, participants mentioned the physical causes of infertility and the impact of infertility on their physical condition. In the psychological sub theme, participants mentioned of the life expectancy, depression, fear, aggression, jealousy, loneliness and inferiority. In the beliefs and religious sub theme, participants were satisfied with the outcome, trust in God and accepting the terms, achieving peace through religious programs, and the futility of their vow of luck. In the social-relations sub theme, participants implied the husband support, family support, husband and family reactions to infertility, interaction and communication in the workplace and the person's judgment and opinion as the main themes. Insecurity and instability in life were as an important sub theme in experience of infertile menopausal women. Instability in marriage, forced divorce, husbands baseless excuses, fear of homelessness and financial burden on the family after the husbands deceased, husbands unreasonable excuses and lack of financial assignment from the husbands were the most important teams of the insecurity and instability in life sub theme.

In the sub theme of strategies, participants mention of medical and traditional treatments, taking their husband to marry again, trying to effectively communicate with their husband children and divorcement request of their husband. However, our findings indicate that participants emphasize the important main theme of motherhood. It can be said of the participants the main theme of life is "worthlessness everything to motherhood pleasure" and "agreement to change the all world pleasures with motherhood". Participant No.2, housewife and 55-year-old woman: "I satisfied live alone on a mountain with a kettle and teapot, but I have just a child, if I haven't water, eat or wear, isn't matter. Wish I had a child of my own". Participant No.7, housewife 54years old woman: "when my husband's grandchild was little, her mother and father taught, I handed her with formula. I love her like my own child. When she told me Mum as the whole world give to me. I was young and happy. Now my little girl grew up and her mother let not me to see my girl! I'll die of sorrow".

Table 2 Experience with menopausal infertile women

\begin{tabular}{|c|c|c|c|}
\hline Main theme & Sub themes & \multicolumn{2}{|l|}{ Meaning units } \\
\hline & \multirow[t]{2}{*}{ Physical factors } & Infertility cause & $\begin{array}{l}\text { Incompletely physical maturity } \\
\text { Childhood disease }\end{array}$ \\
\hline & & \multicolumn{2}{|c|}{ Physical impact of infertility } \\
\hline & & & Hoped new therapies \\
\hline & & Life expectancy & Hopelessness for the lack of detection \\
\hline & Psychological factors & & Hopelessness for menopause \\
\hline & & Inferiority & \\
\hline & & Fear & \\
\hline & & Depression & \\
\hline & Beliefs and religious factors & Satisfactory of d & \\
\hline & & Vow & \\
\hline \multirow{11}{*}{ Motherhood } & & Blaming the fam & \\
\hline & Social relations & \multicolumn{2}{|c|}{ The husband's excuses } \\
\hline & & \multicolumn{2}{|c|}{ Person's judgment } \\
\hline & & Marital life & \\
\hline & Insecurity and instability in life & \multicolumn{2}{|c|}{ Lack of financial granting and economic problems } \\
\hline & & \multicolumn{2}{|l|}{ Uncertain future } \\
\hline & \multirow{5}{*}{ Strategies } & & Effects of treatment \\
\hline & & Treatment & Side effects of treatment \\
\hline & & & Barriers to treatment \\
\hline & & \multicolumn{2}{|c|}{ Initiative partner to remarry } \\
\hline & & \multicolumn{2}{|c|}{ Establish a good relationship with husband children } \\
\hline
\end{tabular}


Table 3 Meaning unit and statements in menopausal infertile women

\begin{tabular}{|c|c|c|}
\hline Meaning Unit & & Statements \\
\hline \multirow{2}{*}{ Infertility cause } & $\begin{array}{l}\text { Incompletely } \\
\text { physical maturity }\end{array}$ & $\begin{array}{l}\text { Participant No. 2, housewife, } 55 \text { years old:" I never have a menstrual period. My doctor } \\
\text { told me: my uterus hasn't grown and as a uterus of little girl. For this reason I didn't } \\
\text { pregnant". }\end{array}$ \\
\hline & Childhood disease & $\begin{array}{l}\text { Participant No. 2, housewife, } 55 \text { years old:" When I was young, my neck was swollen. } \\
\text { I think we're saying the mumps. I didn't properly cure. My doctors told me: this disease } \\
\text { had been the causes of my infertility". }\end{array}$ \\
\hline $\begin{array}{l}\text { Physical impact of } \\
\text { infertility }\end{array}$ & \multicolumn{2}{|c|}{$\begin{array}{l}\text { Participant No. 12, housewife, } 57 \text { years old:" I hadn't regular menstrual period, like other women, dirty blood } \\
\text { collected in my body. I feel pain in every part of my body. I've got heart and lung disease. Childbearing women } \\
\text { haven't my disease. They are healthier in comparing with me". }\end{array}$} \\
\hline
\end{tabular}
Hoped new therapies embryo for infertile women. I know a woman, 60 years old. Her doctors implanted a baby for her. Her child was born three months ago. She is 5 years older than I am.

Hopelessness for the

Life expectancy

Fear

Depression

Satisfactory of destiny

Vow

Blaming the family

The husband's excuses

Person's judgment lack of detection

Hopelessness for menopause a bite of food".
Participant No. 3, marketer, 50 years old: "My husband says: if there is a beneficial treatment, I cost for you again. But I need to know, is anyone with my problem become pregnant. There is anyone like me".

Participant No. 1, housewife, 56 years old:"If I wasn’t menopause, I could've hoped. Everything has changed and all infertile women were treated. Except I was unlucky and got an early menopause. When a woman becomes menopause, doctors cannot perform anything for her. Menopause means everything ended".

Participant No. 8, housewife, 52 years old:"I know I'm infertile, but I had to marry again. If I haven't a husband, I have to return my brother home and servant for my brother's wife. So, I have to wash their clothes, and waiting for

Participant No. 4, housewife, 52 years old:" when I was 16 years old, the doctor examined me. He told me: I am healthy. Doctor examined my husband's and said: your husband never has a child. My doctor said to me: your husband is sterile. If you wait for your husband, you'll never be the mother. But my fear of my husband, so I did tell anyone that my husband is the cause of my infertility ".

Participant No. 12, housewife, 59 years old:" When my doctor told: my treatment is useless, I cried a lot of. I decided to burn me. What is the benefit my survival? I'll be old and alone. In the future, I will be more disabled and I'm not anyone to give me a glass of water".

Participant No. 7, housewife, 54 years old:”Always I say: My God I'm pleased for your pleased. This world isn't worth. Give me a good place in another world".

Participant No. 6, housewife, 48 years old:” It was my own fault that I was infertile. Since I was a young marriage. I'm Ashamed that pregnant soon. I votive haven't pregnant three years. Since it's a vow I'm infertile".

Participant No. 5, tailor, 49 years old: "Some people tell me: Why do you work? Give little your punishment. You haven't children, hardly awake for whom?".

Participant No. 6, housewife, 48 years old:"Sometimes people say my husbands the same things that told me. They tell my husband: why you work hardly. You haven't children. This time, when my husband returns to our home, usually he says nothing, but I can understand all things from his behavior. "

Participant No. 3, marketer, 50 years old: "People say to me: because my husband has children, therefore, he doesn't burn for me and he doesn't my treatment properly". 


\section{Meaning Unit}

Statements
Marital life

Lack of financial granting and economic problems

Uncertain future
Participant No. 13, retired, employee, 52 years old. "In all the 25 years that I lived with my husband, I'm always waiting, my husband tells me: I want to get a wife. Two years ago, my husband remarried. If my husband was the cause of infertility, I did not leave my husband. But my husband got married when I'm old".

Participant No. 17, housewife, 60 years old:"8 years after marriage, my husband remarried. Always men are faithless. But if a man is infertile, his wife will be with him and will tolerate suffering".

Participant No. 4, housewife, 56 years old:"My husband says: because you haven't children, I can give anything from my fortune for you. If you born children of my own blood, I will assign my house to you".

Participant No. 7. Housewife, 54 years old:" If my husband died, his children, they will evict me from my husband's home".

Effects of treatmen

Participant No. 15, housewife, 57 years old:"Every time I went to the doctor to give me injections, I had menstruation and all pains went through my body. Dirty blood cause diseases and woman will be feeling bad".

Side effects of treatment

Participant No. 10, barber, 51 years old:"My heart hurts. I feel all parts of my body are broken away. My doctors say to me: It is because of all those pills that I ate for children".

Husband illiteracy: Participant No. 1, housewife, 56 years old:"My doctors told me: I will be treated in Tehran. My husband loved me as his own. But my husband was illiterate. My husband said to me: If you can treatment here, I would sell all my life for your treatment, but whom we take to Tehran".

Barriers to treatment

Economic problems: Participant No.11, housewife, 55 years old:"If I had the money, I used for embryo implantation. But embryo implantation is expensive". Participant No. 9, housewife, 52 years old:"When we were young, we hadn't money and we are sharing with my husband's parents. Because we're sharing, we afraid of my husband's parents. We couldn't spend money to treat ourselves".

Participant No. 7, housewife, 54 years old:"Infertility is a long story. We may live 100 years. A kid is required to give us a glass of water. When I saw that, my husband hasn't the money to my treat, I voluntary asked him to marry again. I said to my husband: your children are like my children".

Initiative partner to remarry

Participant No. 16, housewife, 57 years old:"I am happy because I took a wife for my husband. Now my husband has a 5 year old daughter. She tells Mom to me. My sister also has infertility. I say to my sister: Take a woman for your husband. Whether your husband's children are your children, too"

Participant No. 14, housewife, 65 years old:"I've endured so much misery. Even I got a wife of my relatives for my husband's son. Because I wanted to have a good relationship with my husband's son. I hope he is closer to me. But it was no avail"
Establish a good relationship with husband children
We searched the English-language medical literature using the international databases including PubMed/Medline, Scopus and ISI Web of Knowledge. As well as Scientific Information Databases (SID), Global Medical Article Limberly (Medlib), Iranian Biomedical Journals (Iran Medex), Iranian Journal Database (Magiran) were explored. Using the Medical Subject Headings (MeSH). We searched "infertility", "post-menopausal", "post-menopausal infertile women" and "experience of living with infertility" including all subheadings. physical factors, psychological factors, beliefs and religion, social relationships, resourcefulness, and the insecurity and instability were the sub-themes in the women with life experience with infertility. 
The Persian keywords were equivalent to their English words and all probable combinations were considered. Moreover, the references of selected citations were hand-searched. All sources were searched with the same strategy and keywords. Several studies have investigated the experience of infertility in couples ${ }^{1}$ and women ${ }^{22}$ who have hope for a future pregnancy by quality and quantity methods. ${ }^{6,23-25}$

Nelson et al., ${ }^{15}$ evaluated the relationship between reproductive history and menopausal symptoms in 436 women $35-47$ years old. ${ }^{15}$ Participants in the study were followed for 14years. There are two key conflicts between our study and the Nelson et al study. We had done a qualitative study. Our study data was collected according participants personal experience. But in Nelson's study used of the quantitative method. Data were collected using standard tools Penn Menopausal Symptoms. ${ }^{15}$ Another major difference between our study and the Nelson et al study is differences in the study population. In our study, all participants were infertile and had never experienced motherhood. But in Nelson et al study, participants in the study experienced a range of non-motherhood to multi-parous women. ${ }^{15}$ Most participants in the current study, believe that experienced more physical and psychological problems than fertile women. Other studies reported that all physical and psychological complication is higher in infertile women in comparison with fertile women. ${ }^{25-28}$ Based our results, the beliefs and religious factors are the important concepts in infertile menopausal women. Consistent with our results a grounded theory study, showed that the religious is a main factor among infertile couples. Therefore, the infertile couples accepted infertility as predetermined God's plan. ${ }^{29}$ Another grounded theory study, investigated the impact of religion on marital fidelity. Researcher of this study reported that religious helped the couples to better control their anger and resolve their issues in a calm and peaceful manner. ${ }^{30}$

Based our results the husband support, family support, and the husband and family reactions to infertility, interaction and communication in the workplace and the person's judgment and opinion were the main sub theme in menopausal infertile women. In another study, infertile women reported the social pressures due to infertility. They complained of the people frequent questions, especially in cases where infertile women experiencing failure treatment. ${ }^{6}$ In the sub theme of strategy, most participants in our study had used medical or traditional treatments. Initiative partner to remarry and establish a good relationship with husbands' children were other strategies that our participants' choice in their lives. So far, different matching methods have been reported in infertile women. Trust in God is discussed as a strategy for infertile women in Islam. ${ }^{6}$ In another study, about half of infertile women were used of spiritual healing and herbal treatment in addition to medical treatment. ${ }^{31}$ Use of complementary medicine in Muslim women infertile is probably due to strong religious beliefs. ${ }^{6}$ Our study has several limitations. The National database have not appropriate of quantity (number of papers have been recorded) and quality (tool). This database does not include all the journals. On the other hand, much of the scientific research in Iran is thesis projects. Unfortunately, there is no comprehensive and coherent national database to cover up the thesis projects.

\section{Conclusion}

Motherhood is the main concept of life experience in menopausal infertile women. Physical factors, psychological factors, beliefs and religion, social relationships, instability in life and strategies are the main sub theme of menopausal infertile women experience. However, we studied the menopausal infertile women with all educational and economic levels to increase the transferability of data; but also, all participants lived in the same culture conditions. Therefore, similar studies are recommended in different cultures.

\section{Acknowledgements}

We express our appreciation and gratitude to the deputy of research of Ilam University of Medical Sciences for supporting this thesis.

\section{Conflict of interest}

The authors declare that they have no competing interests.

\section{References}

1. Tabong PT, Adongo PB. Infertility and childlessness: a qualitative study of the experiences of infertile couples in Northern Ghana. BMC Pregnancy and Childbirth. 2013;13:72.

2. Direkvand-Moghadam A, Delpisheh A, Khosravi A. Epidemiology of female infertility. a review of literature. Biosci Biotech Res Asia. 2013;10(2):559-567.

3. Bhattacharya S, Johnson N, Tijani HA, et al. Female infertility. BMJ Clin Evid. 2010;pii:0819.

4. Mascarenhas MN, Flaxman SR, Boerma T, et al. National, regional and global trends in infertility prevalence since 1990 . a systematic analysis of 277 health surveys. PLoS Med. 2012;9(12):e1001356.

5. Mascarenhas MN, Cheung H, Mathers CD, et al. Measuring infertility in populations: constructing a standard definition for use with demographic and reproductive health surveys. Popul Health Metr. 2012;10(1):10-17.

6. Obeidat H, Hamlan A, Callister L. Missing Motherhood: Jordanian Women's Experiences with Infertility. Advances in Psychiatry. 2014;2014(2014):7.

7. Volgsten H, Skoog Svanberg A, Ekselius L, et al. Risk factors for psychiatric disorders in infertile women and men undergoing in vitro fertilization treatment. Fertil Steril. 2010;93(4):1088-1096.

8. Volgsten H, Ekselius L, Poromaa IS, et al. Personality traits associated with depressive and anxiety disorders in infertile women and men undergoing in vitro fertilization treatment. Acta Obstet Gynecol Scand. 2010;89(1):27-34.

9. Drosdzol A, Skrzypulec V. Evaluation of marital and sexual interactions of Polish infertile couples. J Sex Me. 2009;6(12):3335-3346.

10. Drosdzol A, Skrzypulec V. Depression and anxiety among Polish infertile couples--an evaluative prevalence study. J Psychosom Obstet Gynaecol. 2009;30(1):11-20.

11. Management and Programming Organization for sight population of Iran during the next 20years. Health Medical and Education Medic in Ministry. 1383:66.

12. Reed SD, Ludman EJ, Newton KM, et al. Depressive symptoms and menopausal burden in the midlife. Maturitas. 2009;62(3):306-310.

13. Rapkin AJ. Vasomotor symptoms in menopause: physiologic condition and central nervous system approaches to treatment. Am J Obstet Gynecol. 2007;196(2):97-106.

14. Nagata C, Takatsuka N, Kawakami N, et al. Soy product intake and hot flashes in Japanese women: results from a community-based prospective study. Am J Epidemiol. 2001;153(8):790-793.

15. Nelson DB, Sammel MD, Patterson F, et al. Effects of reproductive history on symptoms of menopause: a brief report. Menopause. 2011;18(10):1143-1148. 
16. Sabia S, Fournier A, Mesrine S, et al. Risk factors for onset of menopausal symptoms: results from a large cohort study. Maturitas. 2008;60(2):108121.

17. Hess R, Olshansky E, Ness R, et al. Pregnancy and birth history influence women's experience of menopause. Menopause. 2008;15(3):435-441.

18. Reid WA. Conceptions of curriculum and paradigms for research: The case of school effectiveness studies. Journal of Curriculum and Supervision. 1997;12(3):212-227.

19. Streubert H, Streubert HJ, Carpenter DR. Qualitative research in nursing 5th ed. USA: Lippincott Williams \& Wilkins; 2011. p. 1-470.

20. Hsieh HF, Shannon SE. Three approaches to qualitative content analysis. Qual Health Res. 2005;15(9):1277-1288.

21. Speziale HJ, Carpenter DR. Qualitative Research in Nursing: Advancing the Humanistic Imperative. 4th ed. USA: Lippincott Williams and Wilkins; 2007. p. 1-447.

22. Paulette F,Sandra LC. Exploring the Long-term impact of female infertility a qualitative analysis of interviews with postmenopausal women who remained childless. The Family Journal. 2013;21(2):180-188

23. Cousineau TM, Domar AD. Psychological impact of infertility. Best Pract Res Clin Obstet Gynaeco. 2007;21(2):293-308.

24. Monga M, Alexandrescu B, Katz S, et al. Impact of infertility on quality of life, marital adjustment, and sexual function. Urology. 2004;63(1):126130 .
25. Cwikel J, Gidron Y, Sheiner E. Psychological interactions with infertility among women. Eur J Obstet Gynecol Reprod Biol. 2004;117(2):126-131.

26. Damti OB, Sarid O, Sheiner E, et al. Stress and distress in infertility among women. Harefuah. 2008;147(3):256-276.

27. An Y, Wang Z, Ji H, et al. Pituitary-adrenal and sympathetic nervous system responses to psychiatric disorders in women undergoing in vitro fertilization treatment. Fertil Steril. 2011;96(2):404-408.

28. Gerrity D. A biopsychosocial theory of infertility. The Family Journal. 2001;9(2):151-158.

29. Latifnejad Roudsari $\mathrm{R}$, Allan $\mathrm{H}$, et al. A qualitative inquiry into the mediating role of religion and spirituality in adjusting marital relationships of infertile women. J Midwifery Reprod Health. 2013;1(1):33-41.

30. Dollahite DC, Lambert NM. Forsaking all others: how religious involvement promotes marital fidelity in Christian, Jewish, and Muslim couples. Review of Religious Research. 2007;48(3):290-307.

31. Bardaweel SK, Shehadeh M, Suaifan GA, et al. Complementary and alternative medicine utilization by a sample of infertile couples in Jordan for infertility treatment: clinics-based survey. BMC Complement Altern Med. 2013;13:35. 